\title{
Klostermedizin - Heilwissen aus der Antike bis heute
}

Johannes Gottfried Mayer

\section{ZUSAMMENFASSUNG}

Europäische Klöster waren vom 8. bis zum 12. Jahrhundert wesentlich für die medizinische Versorgung. Antike Schriften dienten als Vorbild und prägten das heilkundliche Wissen. Die Klostermedizin umfasst dabei eine medizingeschichtliche Epo- che, die auf die Regula Benedicti des Heiligen Benedikt zurückgeht. Die Klöster entwickelten sich zum Ort medizinischer Versorgung. Die zentralen heilkundlichen Ansätze der Klostermedizin waren die Humoralpathologie und die vier Primärqualitäten. Abhängig von den Primärqualitäten einer Erkrankung setzt man in der Klostermedizin Heilpflanzen mit jeweils gegenteiligen Qualitäten zur Behandlung ein.
Überlieferte Schriften gewähren uns heutzutage Einblicke in die Klostermedizin, z. B. das Lorscher Arzneibuch. Eine auf den ersten Blick recht befremdliche Rezeptur darin fand schon vor Jahren das Interesse der Forschung. Demnach soll man in eine tiefe Wunde am Unterschenkel eine Mischung aus Käse, Schafdung und Honig einbringen. Theoretische pharmakologische Überlegungen ergaben, dass dieses Mittel jedoch tatsächlich wie eine Art Antibiotikum wirken könnte [1].

\section{Die Anfänge der Klostermedizin}

Der Begriff der Klostermedizin stammt aus der Medizingeschichte und bezeichnet eine Epoche der europäischen Medizin, die in ihrer Hauptphase etwa das 8. bis 12. Jahrhundert n. Chr. umfasst. Ihr Ursprung geht auf die „Regula Benedicti“ des Heiligen Benedikt von Nursia zurück, dem Begründer des Benediktinerordens. $\mathrm{Er}$ hat dieses Regelwerk in 73 Kapiteln für das um 529 gegründete Kloster Monte Cassino verfasst. Kapitel 36 legt fest, dass jedes Kloster über eine Krankenstation und einen geschulten Pfleger verfügen solle. Papst Gregor I. (540-604) erklärte die Regel Benedikts zum Vorbild für die Klöster der katholischen Kirche. Die Mönche begannen deshalb, sich mit der medizinischen Literatur der Antike zu befassen. Cassiodor Senator (ca. 485-580) gründete im hohen Alter von 70 Jahren das Kloster Vivarium in Kalabrien. Er empfahl in seinen „Institutiones“ das Studium der Schriften von Hippokrates, Dioskurides, Galen und anderen griechischen Ärzten, um die Heilkunde in den Klöstern zu bewahren. Als der fränkische König und Kaiser Karl der Große (747/748-814) sein Reich nach dem Vorbild des römischen Imperiums reformieren wollte, übertrug er den Klöstern neben vielen anderen Aufgaben auch die medizinische Versorgung.

\section{Die ersten Dokumente der Klostermedizin}

Das Lorscher Arzneibuch war das wichtigste Dokument in der ersten Phase der Klostermedizin im Frankenreich. Es wurde um 790 / 795 im östlich von Worms gelegenen Kloster Lorsch ( $\mathbf{A b b} \mathbf{1}$ ) geschrieben [2]. Die Handschrift wurde 2013 in das Weltdokumentenerbe der UNESCO aufgenommen. Die bemerkenswerte Einleitung rechtfertigt die Medizin in der Christenheit. Darin sind auch die „Institutiones“ von Cassiodor zitiert. Der Hauptteil umfasst fünf Rezeptsammlungen, die vorwiegend aus antiken Quellen stammen. Über 600 verschiedene Pflanzenarten kommen als Ingredienzien zum Einsatz. Die am häufigsten genannte Pflanze ist der Fenchel eine der beliebtesten Arzneipflanzen der ganzen Klostermedizin. Überraschend häufig finden sich aber auch Gewürze aus Asien, allen voran Ingwer und Pfeffer sowie Harze aus Arabien wie Myrrhe, Mastix, Weihrauch oder Gummi arabicum - das Harz verschiedener Acacia-Arten.

Ein weiteres Dokument der ersten Phase der Klostermedizin ist der St. Galler Klosterplan aus der Zeit zwischen 819 und 826. Dieser große Plan eines idealen Klosters zeigt im oberen (östlichen) Teil einen ganzen Krankenkomplex mit Spital, Arzthaus und Kräutergarten. Im Haus des Mönchsarztes waren auch die Schwerkranken untergebracht, damit er sie umgehend versorgen konnte [3]. Dieser Klosterplan entstand im Inselkloster Reichenau im Bodensee. Von 838 bis zu seinem Tod im Jahr 849 unterstand das Kloster Walahfrid Strabo. Der Abt war einer der talentiertesten Dichter seiner Zeit. Von ihm stammt auch ein wunderbares Gedicht über einen Kräutergarten, der „Liber de cultura hortorum“ oder kurz „Hortulus“ [4]. Das Gedicht beschreibt 24 Pflanzen, wobei er auch die wichtigsten Anwendungen nennt. Hier finden sich Schlafmohn und Heilziest, Wermut und Andorn, Fenchel, Salbei, Rose, Lilie, Wermut, Weinraute und Eberraute (s. Porträt S. 283), 


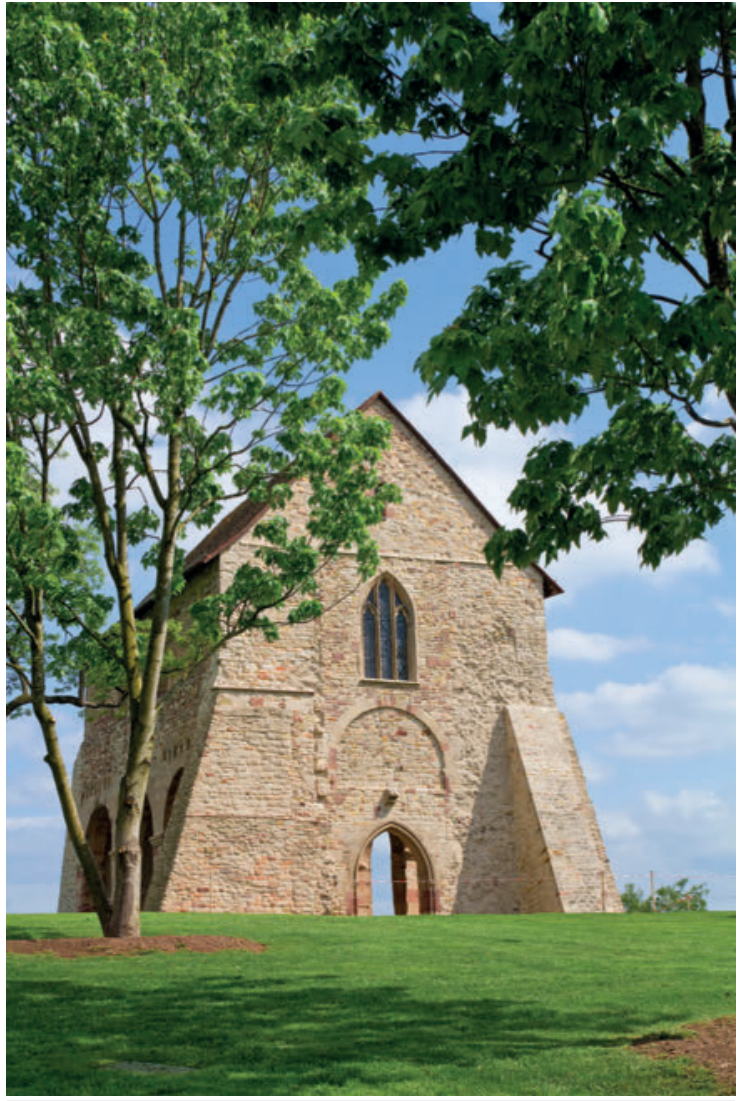

- Abb. 1 Kloster Lorsch: Das Lorscher Arzneibuch ist eine der wichtigsten Schriften der Klostermedizin. In 5 Rezeptsammlungen sind über 600 Heilpflanzen beschrieben. Foto: Adobe Stock / Elisabeth

aber auch Flaschenkürbis und Melone, Minze und Poleiminze, Kerbel und Liebstöckel, Sellerie und Rettich. Letztere nutzte man nicht nur in der Küche, sondern auch als Heilpflanzen. Melisse, Kamille, Thymian, Rosmarin und Lavendel sind in diesem Gedicht nicht genannt. Im Lorscher Arzneibuch spielen sie auch keine große Rolle oder fehlen ganz.

\section{Theorie und Krankheitslehre}

Die erste Phase der Klostermedizin hat keine größeren Texte zu der Entstehung von Erkrankungen und zur Theorie der Humoralpathologie und ihren vier Primärqualitäten - warm, kalt, trocken, feucht - hervorgebracht. Die Schriften dieser Zeit befassen sich vorwiegend mit den Heilwirkungen der Pflanzen oder liefern entsprechende Rezepte. Das änderte sich im 11. Jahrhundert mit Konstantinus Africanus (gest. 1087). Der aus Nordafrika stammende Araber oder Berber hatte Medizin studiert und kam schließlich nach Salerno in Süditalien an die dortige Medizinschule. 1078 trat er in das vom Heiligen Benedikt gegründete Kloster Monte Cassino ein. Er übersetzte zahlreiche medizinische Schriften aus dem Arabischen ins Lateinische.
Seine Übersetzungen führten zu einem enormen Aufschwung der Medizin in Europa. Salerno entwickelte sich zur wichtigsten ärztlichen Ausbildungsstätte in ganz Europa. Indirekt dürften die Werke Constantins auch Hildegard von Bingen beeinflusst haben, denn sie hat sich der Humoralpathologie angeschlossen - wenn auch in eigener Interpretation. Von nun an richtete sich die Krankheitslehre und Therapie in der Klostermedizin nach der Humoralpathologie - der Viersäftelehre der Antike. Durch die Übersetzungen von Konstantinus Africanus rückte die Humoralpathologie der Antike ins Zentrum der Klostermedizin.

\section{QUELLEN \\ Wichtige Werke der Klostermedizin}

Das meistgelesene Werk der Klostermedizin war der „Macer floridus“. Das ist ein umfangreiches Gedicht über die Heilkräfte von 77 Arzneipflanzen aus dem ausgehenden 11. Jahrhundert, das der Mönch Odo von Meung in Frankreich bei Orleans schrieb [5]. Die beiden natur- und heilkundlichen Schriften der Hildegard von Bingen (1098-1179 n. Chr.) „Causae et curae" und „Physica“ sind heutzutage erheblich bekannter. Diese Werke verfasste sie gegen Ende der klostermedizinischen Epoche. Zahlreiche Universitätsgründungen im ausgehenden 12. und beginnenden 13. Jahrhundert läuteten eine neue Epoche ein. Denn nun gab es akademisch gebildete Ärzte, die nicht dem Klerus angehörten.

\section{Entwicklung der Humoralpathologie}

Schon vor Hippokrates (ca. 460-370) kam in der griechischen Heilkunde der Gedanke auf, dass sich die Gesundheit des Menschen auf einem harmonischen Gleichgewicht von Gegensätzen gründet. Solche Gegensätze sind nach Alkmaion (ca. 500-420) zum Beispiel warm und kalt, feucht und trocken, süß und sauer, männlich und weiblich, hell und dunkel sowie ruhend und bewegt. Diese Ansicht erinnert stark an das Ying-Yang-Prinzip der chinesischen Medizin. In der weiteren Entwicklung der europäischen Medizin erhielten die beiden Gegensätze warm und kalt sowie feucht und trocken zentrale Bedeutung als die Primärqualitäten der Humoralpathologie.

Der Begriff der Humoralpathologie setzt sich aus den beiden Wörtern "humor“ (= Saft) und Pathologie (= Krankheitslehre) zusammen. Die Grundprinzipien wurden erstmals in der Schrift „Über die Natur des Menschen" dargestellt. Diese geht auf den Arzt Polybos, den Schwiegersohn des Hippokrates, zurück. Polybos ging davon aus, dass der menschliche Organismus von vier Säften bestimmt wird: Blut (Sanguis), Schleim (Phlegma), Gelber Galle (Cholera) und Schwarzer Galle (Melancholia). 
Aber erst in den Schriften des Galen von Pergamon (127 / 29-ca. 200 n. Chr.) findet sich das vollständige System der Humoralpathologie mit den vier Primärqualitäten warm-kalt und feucht-trocken ( $\triangleright$ Tab. 1).

\section{Die Krankheitslehre der Humoralpathologie}

Das System umfasste nicht nur die Körpersäfte und Organe des Menschen, sondern auch die einzelnen Erkrankungen und die entsprechenden Heilmittel.

Es lässt sich am besten an konkreten Beispielen darstellen, etwa an einem Schnupfen. In der Humoralpathologie ist die Grundursache für einen Schnupfen ein Übermaß an Phlegma (Schleim). Phlegma ist kalt und feucht und sammelt sich beim gesunden Menschen vornehmlich im Gehirn an ( $\triangleright$ Tab. 1$)$. Ist der Mensch innerlich kalt, steigt zu viel Phlegma in den Kopf. Das kann der Fall sein, weil seine Organe träge arbeiten, durch äußere klimatische Einflüsse, wie sie vor allem im Winterhalbjahr herrschen, oder durch Überanstrengung. Das resultiert in Druck und damit Kopfschmerz. Das überflüssige Phlegma kann durch innere oder äußerliche Einflüsse - etwa durch Kälte oder Hitze - verändert werden. Es entsteht eine pathogene Flüssigkeit, welche die Atemwege in der Nase und im Rachen verstopft. Man entwickelt einen Schnupfen bzw. einen Katarrh der Atemwege. Der Körper versucht nun, diese Flüssigkeit durch Niesen und Husten auszuscheiden. Oft tränen auch die Augen.

In der Schule von Salerno werden um 1125 die Zeichen eines Erkältungsschnupfens aus kalter Ursache so beschrieben: „fahle Gesichtsfarbe, Tränen werden als kalt empfunden und erkälten die Haut des Gesichts, der Leidende fühlt Kälte tief im Leib, und er gibt an, er sei durch abkühlende Speisen und Tränke und durch die Kälte der Luft in Befinden und Komplexion (Konstitution) verändert worden“ [6].

Zwischen 1150 und 1160 schildert Hildegard von Bingen in ihrer Schrift „Causae et curae“ dies ähnlich: Sind die

- Tab. 1 Grundprinzipien der Humoralpathologie.

\begin{tabular}{|c|l|l|l|}
\hline Primärqualitäten & Elemente & Körpersaft & Organ \\
\hline warm und feucht & Luft & Blut/Sanguis & Herz \\
\hline warm und trocken & Feuer & $\begin{array}{l}\text { gelbe Galle } / \\
\text { Cholera }\end{array}$ & Leber \\
\hline kalt und trocken & Erde & $\begin{array}{l}\text { schwarze } \\
\text { Galle } /\end{array}$ & Milz \\
Melancholia & \\
\hline kalt und feucht & Wasser & $\begin{array}{l}\text { Schleim/ } \\
\text { Phlegma }\end{array}$ & Gehirn \\
\hline
\end{tabular}

Verdauungsorgane kalt und schwach, senden sie ihren kalten, feuchten Brodem in das Gehirn. Dieser Schleim wird zu einem Gift und zieht die Krankheitskeime der erkrankten Säfte zusammen, „so dass sie unter Schmerzen durch die Nase und den Rachen ausgeschieden werden".

\section{HINTERGRUND \\ Wie grenzt sich die Klostermedizin von der Natur- heilkunde ab? \\ Fast 1000 Jahre trennen die Entwicklung der Natur- heilkunde und der Klostermedizin. Die Naturheil- kunde entwickelte sich erst im 19. Jahrhundert vorwiegend im deutschen Sprachraum. Zunächst war sie lediglich eine Umbenennung der Hydropa- thie, die vor allem Nicht-Ärzte betrieben. Diese Methode umfasste in erster Linie innerliche und äußerliche Anwendungen von kaltem Wasser, aber auch Bewegungs- und Ernährungskuren sowie eine entsprechende natürliche Lebensweise zur Gesund- erhaltung. Erst nach 1890 wurden durch den „Was- serdoktor und Kräuterpfarrer“ Sebastian Kneipp (1821-1897) einige Heilkräuter Teil der Naturheil- kunde. Heute werden die echten Naturheilverfahren weitgehend mit den 5 Säulen (Wasser, Pflanzen, Bewegung, Ernährung, Balance) gleichgesetzt, die man Kneipp zuschreibt. Die Naturheilkunde im Sinne einer traditionellen europäischen Medizin schließt aber auch weitere Verfahren wie Balneologie, auslei- tende Verfahren und Lichttherapie mit ein. Prof. Dr. Dr. Bernhard Uehleke, Berlin}

\section{Therapie mit vier Primärqualitäten}

Einen Schnupfen betrachtete man als eine Erkrankung, die durch übermäßiges kaltes und feuchtes Phlegma entsteht. Um das zu behandeln, benötigte man wärmende und trocknende Arzneimittel. Mehr als die Hälfte aller Arzneipflanzen werden in den verschiedenen Kräuterbüchern als wärmend und trocknend bezeichnet. Wärmende Pflanzen sind zum Beispiel Fenchel, Salbei, Beifuß oder Wermut. Kalte Pflanzen sind dagegen Spitzwegeich, Rose, Schierling oder Schlafmohn (Opium). Entscheidend ist die Wirkung, die eine Droge im menschlichen Körper erzeugt. Neben wärmenden, trocknenden und kühlenden Mitteln gibt es auch befeuchtende.

- Wärmend umfasst ein großes Spektrum an konkreten Wirkungen. Grundsätzlich liegt eine anregende, öffnende Wirkung auf die Schleimhäute in Mund und Rachenraum oder jene im Magen-Darm-Trakt vor. Auch kann die Tätigkeit von Leber und Gallenblase angeregt werden.

- Kühlend Mittel, die eine beruhigende, zusammenziehende und stopfende Wirkung haben. Man setzt sie 
gegen Schmerzen, zur Blutstillung, Wundheilung und Schlafförderung sowie gegen Fieber und Durchfall ein.

- Trocknend bedeutet in einem sehr umfassenden Sinn ausleitend. Es handelt sich nicht nur um diuretische oder aquaretische Eigenschaften, die hier mit inbegriffen sind. Vielmehr heißt trocknend, dass eine Flüssigkeit zum Fließen angeregt wird. Zum Beispiel festsitzender Nasenschleim, Speichel, Magensäfte, Gallensaft, Harn oder Blut (insbesondere auch Menstruationsblut).

- Befeuchtend Dem Körper wird Flüssigkeit zugeführt. Dies geschieht vor allem durch Schleimstoffe (Leinsamen, Bockshornklee) und viele Obstsorten. Mittel, die schnell verderben, werden zu den feuchten Stoffen gezählt, da Keime sich bei Feuchtigkeit gut verbreiten können.

Zur genaueren Einordnung wurden die Primärqualitäten in vier Intensitätsgrade unterteilt. Mittel im ersten Grad sind nur schwach spürbar, Mittel im vierten Grad wirken sehr stark. So sind zum Beispiel wärmende Mittel - wie Pfeffer - in höheren Dosen schon schmerzhaft.

Bei Schnupfen eingesetzte Heilpflanzen sollten also dazu dienen, die Atemwege anzuregen, zu erweitern und den Schleim - das Schnupfensekret - zum Fließen zu bringen. Das sind Wirkungen, die wärmenden und trocknenden Mitteln zugeschrieben wurden. Ganz konkret hat man bei Erkältungskrankheiten Fenchelfrüchte ( $\mathbf{A} \mathbf{b} \mathbf{b} . \mathbf{2}$ ), aber auch Minze-Arten oder Salbei, Heilziest (Betonie), Rettich und Meerrettich empfohlen.

\section{Verdauungs- und Stoffwechselstörungen}

Das Prinzip wird noch deutlicher, wenn man es für pflanzliche Heilmittel gegen Verdauungs- und Stoffwechselstörungen anwendet.

\section{HINTERGRUND}

Stoffwechsel: Nach Galen ein Prozess in 3 Stufen

- Die zerkaute Nahrung wird im Magen und in den oberen Darmabschnitten durch Kochung zu einem Stoff, dem Chylus, umgewandelt, den die Leber weiterverarbeiten kann. Die nötige Wärme für den Kochvorgang liefert die Leber, die tatsächlich das wärmste Organ des menschlichen Körpers ist. Abfallprodukt der Kochung ist der Stuhl.

- In der Leber wird der Chylus zu Blut umgebildet, Abfallstoff ist hier der Urin.

- Über das Herz wird das Blut in das Arteriensystem eingespeist und gelangt von dort in alle Körperregionen, die sich vom Blut ernähren. Die Abfallprodukte dieser letzten Verarbeitung des Blutes werden mit dem Schweiß ausgeschieden.

Verdauung, Stoffwechsel und auch Leben an sich verstand man als Abfolge von Wärmeprozessen (Kasten). Schwere Krankheiten, Alterung und Sterben sind somit Prozesse der Abkühlung und Erkaltung. Störungen und Krankheiten wurden deshalb meist auf einen Mangel an Wärme zurückgeführt. Dieser Mangel führt zum Beispiel zu einer trägen Verdauung. Der Speisebrei wird nicht mehr genügend im Magen gekocht. Auch in diesem Fall

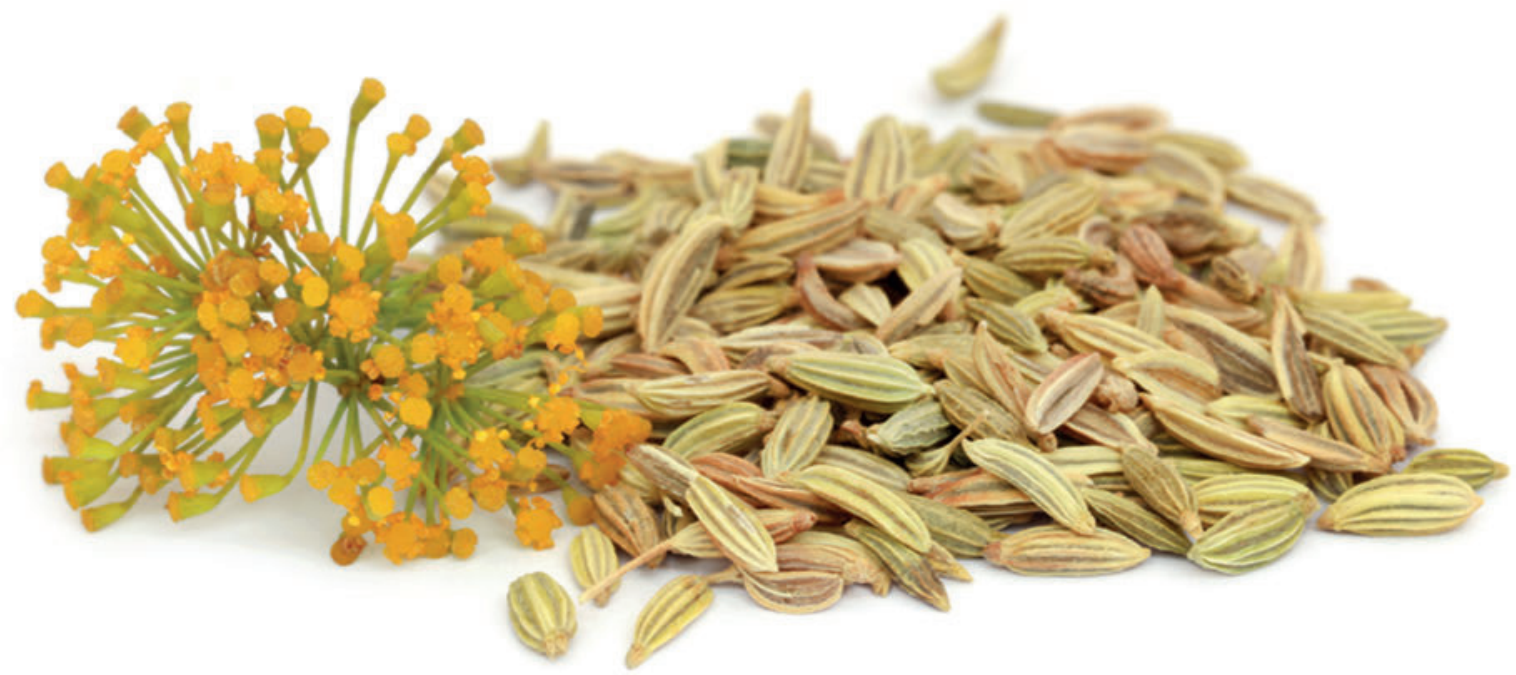

Abb. 2 Fenchelsamen und -blüten. Die Pflanze gilt in der Klostermedizin als wärmend und wird z.B. bei Erkältungen eingesetzt. Foto: Adobe Stock/Swapan 
konnte man durch wärmende Pflanzen Abhilfe schaffen. Häufig setzte man dazu Wermut, Beifuß oder Andorn ein. Aber auch Kümmel, Fenchel, Dill oder Liebstöckel galten als wärmend und geeignet.

Durchfall betrachtete man in der Regel als Überfunktion. Der Organismus galt als überhitzt, und man musste kühlende und trocknende Mittel geben. Bei besonders starken Durchfällen setzte man Opium ein, das als sehr stark kühlend und trocknend eingestuft wurde; ansonsten auch Wegerich, Eibisch und Rose.

\section{Inhaltsstoffe bestimmen die Primärqualitäten mit}

Auf den ersten Blick ist es erstaunlich, dass die moderne Phytotherapie, die Krankheitserreger wie Bakterien und Viren kennt, bei vielen Erkrankungen dieselben Arzneipflanzen verwendet wie die Humoralpathologie bzw. Klostermedizin. Doch bei genauerer Betrachtung fällt auf, dass die Bewertung der Pflanzen nach den Primärqualitäten mit den Inhaltsstoffen zusammenhängt. Pflanzen, die vor allem durch ihr ätherisches Öl wirken, wie Fenchel oder Kümmel, Thymian oder Rosmarin, ordnete man als warm und trocken im 2. oder 3. Wirkungsgrad ein. Ganz ähnlich auch die Bitterstoffdrogen. Zu den ganz warmen Mitteln gehörte der Pfeffer. Er galt als warm und trocken im 4. Grad; Ingwer als warm und feucht im 3. Grad. Die Schleimdrogen wie Leinsamen, Eibischwurzel oder Wegerichblätter galten als kalt und befeuchtend im 1. oder 2. Grad. Toxische Pflanzen mit hohem Alkaloidgehalt wie Schlafmohn, Bilsenkraut oder Schierling bezeichnete man als kalt und trocken im 3 . oder 4. Grad. Keine eindeutige Richtung ließ sich bei den Gerbstoffdrogen erkennen. Der Theorie zufolge müssten sie kühlend sein. Es lassen sich gewisse Beziehungen zwischen der Einschätzung der Klostermedizin und der modernen Phytotherapie ziehen. So lässt sich manches aus der Klostermedizin lernen.

\section{Arzneimittel: die richtige Zubereitung}

In der Epoche der Klostermedizin war der Kräutertee, wie wir ihn heute kennen, ein selten empfohlenes Mittel. An erster Stelle stand die Verabreichung in Wein, entweder als Kaltauszug oder gekocht. Bei vielen Pflanzen erzielte man damit eine höhere Wirkung, da sich manche Stoffe, zum Beispiel ätherisches Öl, in Alkohol besser lösen. An zweiter Stelle standen Honigmittel in fester oder flüssiger Form. Auf diese Weise hat man ein haltbares Mittel hergestellt. Bei Atemwegserkrankungen, Magen- und Hautproblemen hat auch der Honig an sich eine positive Wirkung. Interessant ist das Fenchel-Kapitel im Gartengedicht „Hortulus“ von Walahfrid Strabo: Er hat bei Magenproblemen empfohlen, Fenchel in Ziegenmilch zu kochen. Das könnte tatsächlich sinnvoller sein, als in Wasser, denn ätherisches Öl löst sich bekanntlich besser in Fett als in Wasser. Gegen Husten sollte Fenchel in Wein eingenommen werden. Der Alkohol könnte hierbei die Verfügbarkeit von Wirkstoffen verbessern.

\section{Heute}

Die Prinzipien der Klostermedizin sind nie restlos verloren gegangen. In der Unani-Medizin, die von Pakistan über Nordindien bis nach Westchina vorwiegend von der moslemischen Bevölkerung dieser Länder praktiziert wird, lebt die Medizin, die Galen von Pergamon propagiert und Ibn Sina (Avicenna) weiterentwickelt hat, bis heute weiter. Ihre Bedeutung in Asien ist nicht geringer als Ajurveda. „Unani“ heißt auf Arabisch „ionisch“ und bedeutet letztlich so viel wie "griechische Medizin“.

\section{Erstveröffentlichung}

Dieser Beitrag ist dem Themenheft „Klostermedizin“ der Deutschen Heilpraktiker Zeitschrift 4/2018 entnommen (Karl F. Haug Verlag in Georg Thieme Verlag KG, Stuttgart).

Dr. Johannes Gottfried Mayer

\section{Über den Autor}

\begin{abstract}
Johannes Gottfried Mayer (1953-2019) war Medizin- und Pharmaziehistoriker. Er unterrichtete an den Universitäten Würzburg (Medizingeschichte und Ethik) und Erlangen-Nürnberg (Pharmaziegeschichte). Er war Gründungsmitglied der Forschergruppe Klostermedizin und seit 2009 Leiter der Forschergruppe. Ihr Ziel ist es, einzelne, fast vergessenen Arzneipflanzen, wie Andorn, Heilziest oder den Königsfarn zu untersuchen, aber auch sinnvolle Arzneiformen zu testen und anzuwenden und alte Methoden wie die Fastenpraxis in den Klöstern des Mittelalters wiederzuentdecken.

Auch in der Zeitschrift für Phytotherapie hat Johannes G. Mayer zahlreiche Beiträge publiziert. Wir möchten mit diesem Nachdruck an sein Wirken für die Phytotherapie erinnern.
\end{abstract}

\section{Literatur}

[1] Schneider E. Traditionelle pflanzliche Arzneimittel. Probleme des Wirkungsnachweises. Z Phytother 2001; 22: 241-245, bes. S. 243

[2] Stoll U. Das ,Lorscher Arzneibuch'. Ein medizinisches Kompendium des 8. Jahrhunderts (Codex bambergensis medicinalis 1). Text Übersetzung und Fachglossar (= Sudhoffs Archiv, Beiheft 28). Stuttgart: Franz Steiner Verlag; 1992

[3] Schedl B. Der Plan von St. Gallen. Ein Modell europäischer Klosterkultur. Wien: Böhlau; 2014

[4] Stoffler HD, Hrsg. Der Hortulus des Walahfrid Strabo. Aus dem Kräutergarten des Klosters Reichenau. Mit einem Beitrag von Theodor Fehrenbach, Sigmaringen: Thorbecke; 1978

[5] Mayer JG, Goehl K. Kräuterbuch der Klostermedizin. Der Macer floridus. Darmstadt: Reprint Verlag Leipzig; 2013

[6] Johannes Platearius. Curae. Nosologie der Schule von Salerno. Verfasst im 2. Drittel des 12. Jahrhunderts. Übersetzung: Konrad Goehl. Baden-Baden: Deutscher Wissenschaftsverlag (in Vorbereitung) 